\section{Circularly Polarized Patch Antenna Array Fed by Coplanar Waveguide}

\author{
I-Jen Chen, Chung-Shao Huang, and Powen Hsu
}

\begin{abstract}
A novel design of a circularly polarized linear patch antenna array fed by a coplanar waveguide (CPW) is presented. The array elements are placed in the direction transverse to the feeding CPW line and are excited by a couple of $100 \Omega$ slotlines, which are combined to form the $50 \Omega$ feeding CPW. The circularly polarized radiation is obtained by placing similar perturbation segments to each of the array elements. The simulated and measured axial ratios, return losses, and radiation patterns are presented for an $1 \times 2$ and an $1 \times 4$ arrays with good results. The proposed feeding structure can be extended to design a two dimensional array with good front-to-back ratio on a single substrate with only one straight CPW line.
\end{abstract}

Index Terms-Antenna arrays, circular polarization, coplanar waveguide (CPW), microstrip antenna.

\section{INTRODUCTION}

Planar antenna arrays have been used for microwave and millimeter-wave applications for many years, especially in mobile communications where system design requires low profile, light weight, and high directivity. Many planar antenna arrays have been designed by using the microstripline feeds, however, only a few works so far have used the coplanar waveguide (CPW) to feed the array. The $\mathrm{CPW}$ has gained increasing popularity in recent years, since it has several advantages over the microstripline, such as low radiation losses, less dispersion, easier integration with solid-state active devices, and the possibility of connecting series and shunt elements to the active devices without the need of via holes through the substrate. Thus it deserves more effort to study the CPW-fed antenna array. Among the previously published papers on the CPW-fed antenna arrays that could be found in the open literature, most were designed for the slot array [1]-[5], two for the slotloop array [6], [7], one for the printed-dipole array [8], and two mentioned briefly about the patch array [9], [10]. In this paper, we propose a simple and efficient method to feed the patch array using the CPW. The array elements used are circular patches. They are aligned in the transverse direction of the feeding CPW. A couple of slotlines are used to excite the array elements. These slotlines are bent and combined to form the CPW. The concept of this T-shaped CPW-to-slotlines feeding structure has also been presented in [5] for excitation of the slot array, however their alignment and excitation of the array elements are quite different from ours.

As compared to the linearly polarized antenna, since the circularly polarized antenna is often used in communication systems such as a high-speed wireless LAN or a wireless access by using high frequencies for suppression of the unwanted multipath signals [11], we also investigate the possibility of generating the circularly polarized radiation by the proposed array. By employing the perturbation method described in [12], we have successfully made the array to radiate circularly polarized waves.

Manuscript received December 13, 2002; revised August 2, 2003. This work was supported in part by the Ministry of Education, Republic of China, under Contract 89-E-FA06-2-4, and in part by the National Science Council, Taiwan, R.O.C., under Contract NSC 91-2219-E-002-015.

The authors are with the Department of Electrical Engineering and Graduate Institute of Communication Engineering, National Taiwan University, Taipe 10617, Taiwan, R.O.C. (e-mail: phsu@cc.ee.ntu.edu.tw).

Digital Object Identifier 10.1109/TAP.2004.829855

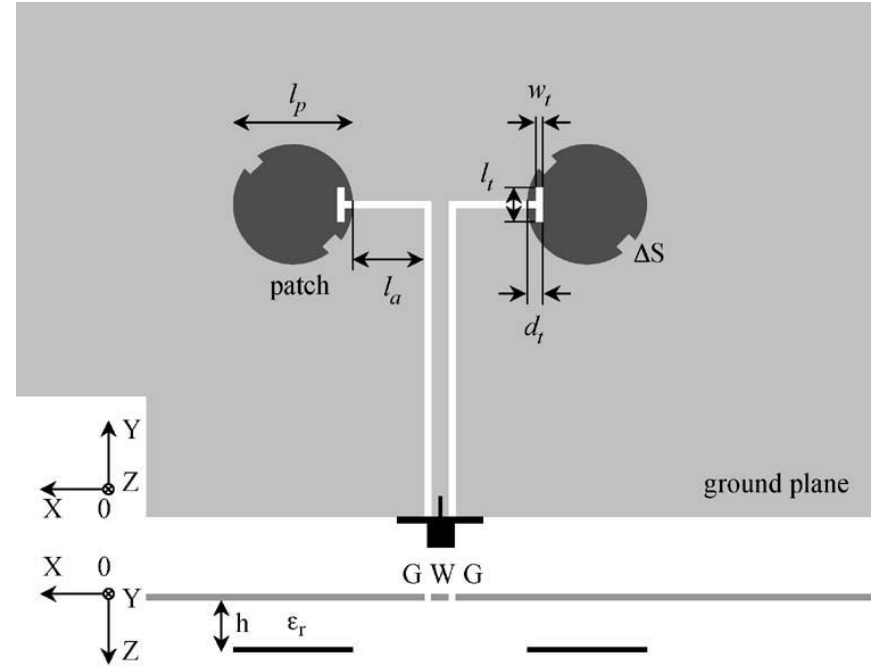

Fig. 1. Side view and view from underneath a $1 \times 2$ circular patch array.

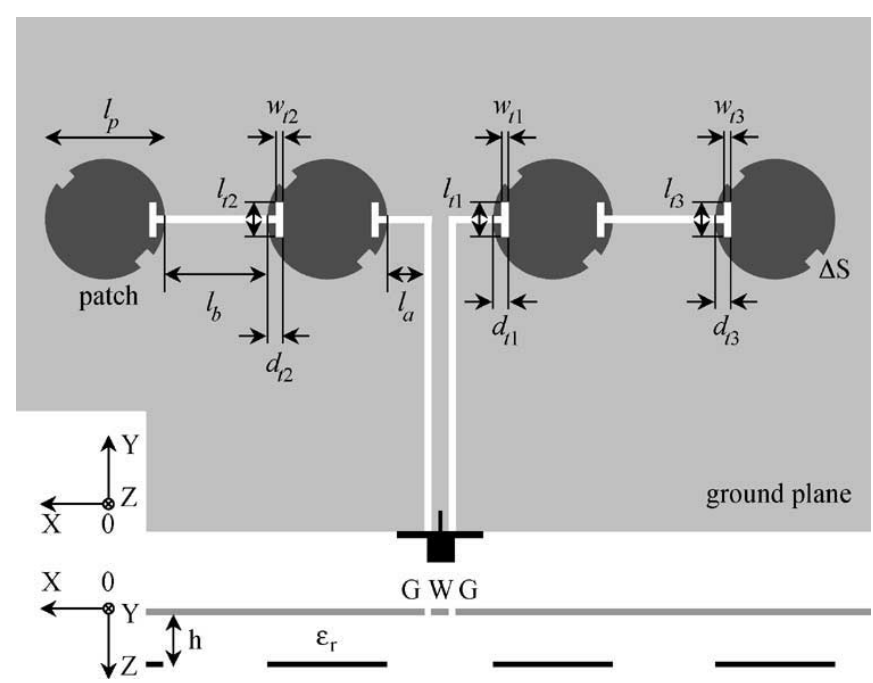

Fig. 2. Side view and view from underneath a $1 \times 4$ circular patch array.

The characteristics of the proposed antenna were clarified by experiments on an $1 \times 2$ and an $1 \times 4$ arrays. Good axial ratios, return losses, and radiation patterns were obtained. One merit of the present design is that it can be extended to form a 2-dimensional (2-D) array by repeating the linear array along the longitudinal direction of the feeding CPW.

\section{DESIGN PROCESS}

The geometries of an $1 \times 2$ and an $1 \times 4$ arrays are shown in Figs. 1 and 2, respectively. Circular patches and the feeding CPW are placed on the opposite sides of the substrate and all the patches are aligned in the transverse direction of the CPW. Slotline sections with transverse end slots are used to connect and excite the patches. Throughout the entire design process, simulations are carried out using the package software Ensemble from Ansoft Corporation, and the FR4 substrate with $h=1.6 \mathrm{~mm}$ and $\varepsilon_{\mathrm{r}}=4.3$ is used for the experimental purpose. The widths of the strip and slot of the CPW are chosen to be 3 and $0.3 \mathrm{~mm}$, respectively, which give a characteristic impedance of $50 \Omega$ for the CPW. The width of the slotline is kept at $0.3 \mathrm{~mm}$ such that its 


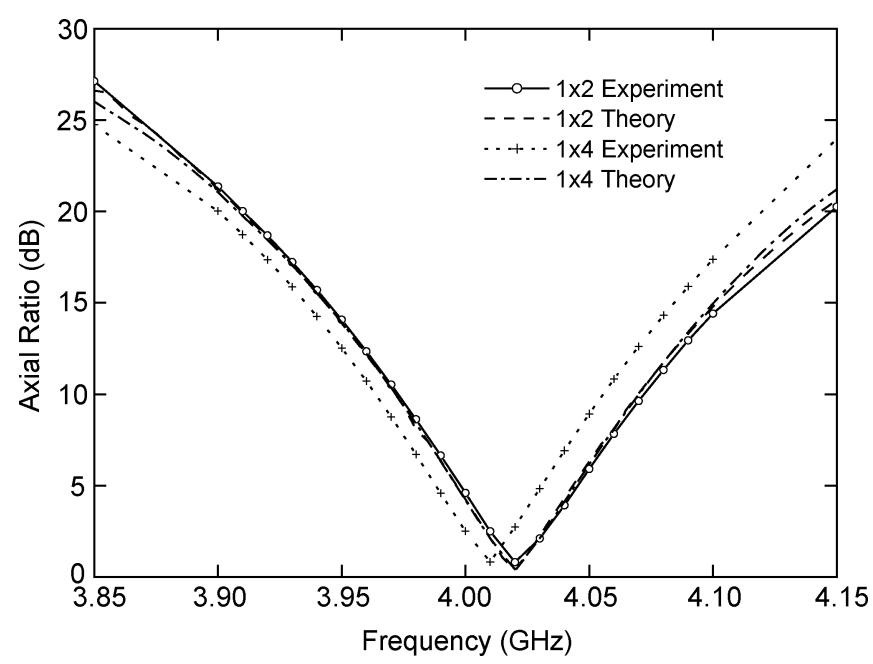

Fig. 3. Measured and simulated axial ratios against frequency.

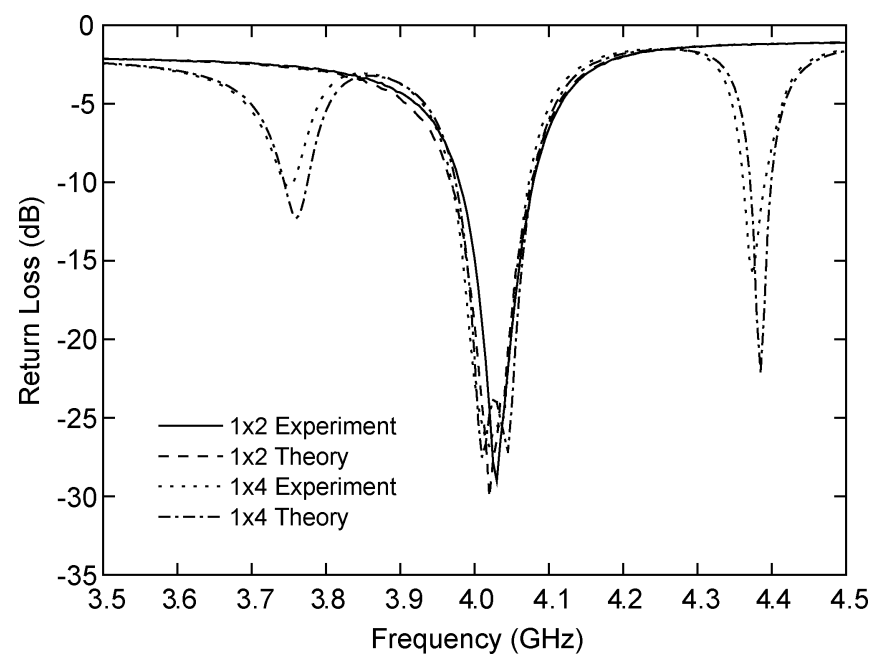

Fig. 4. Measured and simulated return losses against frequency.

characteristic impedance is equal to $100 \Omega$ and the CPW-to-slotlines transition is matched. For simplicity, the widths of all the transverse end slots $w_{t}$ 's are also set at $0.3 \mathrm{~mm}$.

To begin our design, $d_{t}, l_{t}$, and $l_{p}$ in Fig. 1 are chosen to be 2, 6, and $20 \mathrm{~mm}$, respectively, which correspond to a patch resonance at $4.04 \mathrm{GHz}$. To realize the circularly polarized radiation of the antenna, two perturbed segments $\Delta S=0.60 \%$ of the circular patch area are cut at the two opposite ends of the patch diameter, which is $45^{\circ}$ inclined from the vertical. These perturbed segments will make the dominant mode of the patch separate into two orthogonal components with equal amplitude and $90^{\circ}$ phase difference, which is required for a circular polarization [12]. After the perturbation, the perimeter of the patch becomes longer, the patch resonant frequency is thus lowered to $4.0 \mathrm{GHz}$ accordingly, while the minimum axial ratio frequency is found to be at $4.02 \mathrm{GHz}$. In order to have an optimum design that the patches are resonant at the minimum axial ratio frequency, $d_{t}$ 's and $l_{t}$ 's have to be carefully adjusted. In designing the $1 \times 2$ array, $d_{t}$ is adjusted first to have the patch resonant frequency as close to the minimum axial ratio frequency as possible. Then $l_{t}$ is tuned to have these two frequencies coincide with each other. Because of changes in $d_{t}$ and $l_{t}$, the value of minimum axial ratio now gets higher. To bring it down, we slightly adjust the perturbed segments to $\Delta S=0.65 \%$ of the circular patch area. The final design for the $1 \times 2$ array is $d_{t}=2.3 \mathrm{~mm}$ and $l_{t}=8 \mathrm{~mm}$,

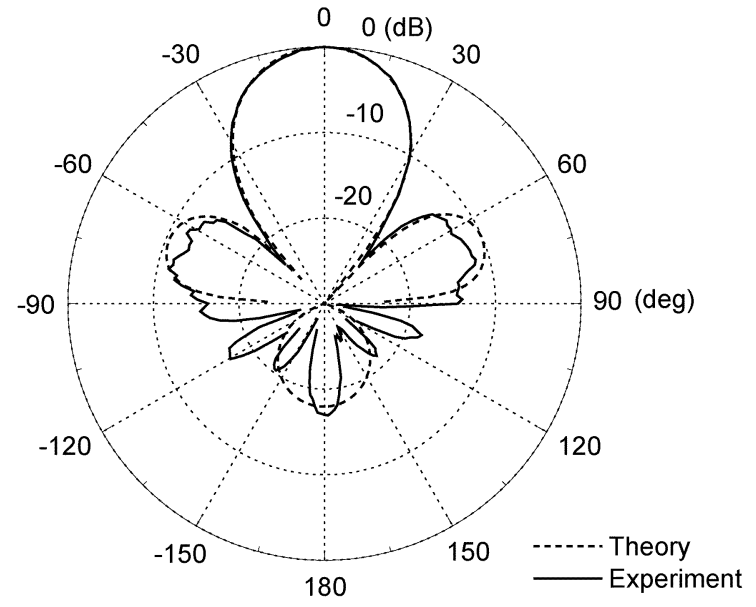

(a)

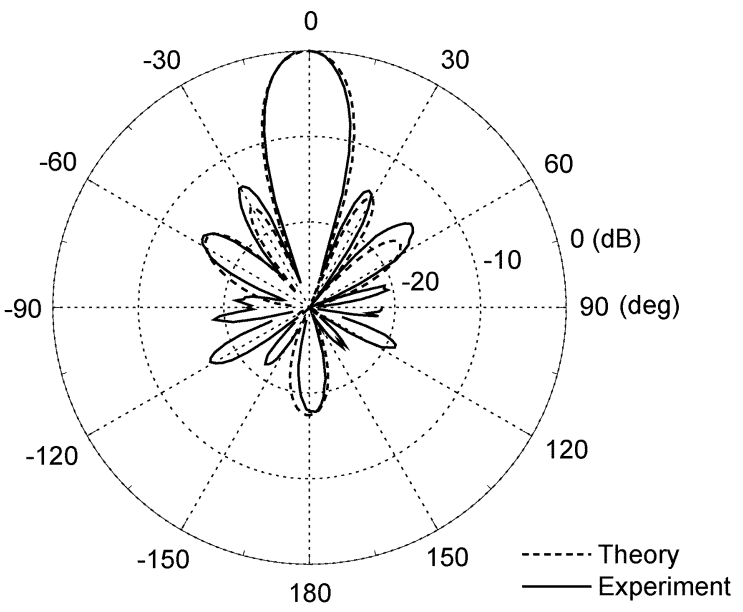

(b)

Fig. 5. Normalized $x-z$ plane RHCP radiation patterns of (a) $1 \times 2$ array and (b) $1 \times 4$ array.

and $l_{a}$ is chosen arbitrarily at $15.7 \mathrm{~mm}$. As for designing the $1 \times 4$ array, the patches away from the CPW are considered first. Since they are the same one-port networks as those in the $1 \times 2$ array, $d_{t 3}$ and $l_{t 3}$ would be expected to have the same dimensions as $d_{t}$ and $l_{t}$ in the $1 \times 2$ array, respectively. The patches near the CPW are individually a two-port network. $d_{t 1}$ and $l_{t 1}$ at the input port are adjusted to have the total input match occurs at the minimum axial ratio frequency, while $d_{t 2}$ and $l_{t 2}$ at the output port are adjusted to have $-3 \mathrm{~dB}$ transmission at that port at the same frequency. Thus, a $1 \times 4$ uniformly excited array is obtained. The length $l_{b}$ is chosen such that every patch is excited in the same phase, while length $l_{a}$ can be arbitrary since it is the length of the feeding slotline section. We choose $l_{a}$ to form an equi-spaced array. The final design of this $1 \times 4$ array is $d_{t 1}=2.5 \mathrm{~mm}, l_{t 1}=8.5 \mathrm{~mm}$, $d_{t 2}=1.8 \mathrm{~mm}, l_{t 2}=5.5 \mathrm{~mm}, d_{t 3}=2.3 \mathrm{~mm}, l_{t 3}=8 \mathrm{~mm}, l_{b}=$ $35.0 \mathrm{~mm}$, and $l_{a}=15.7 \mathrm{~mm}$.

It should be noted that, since the above arrays are small and, except for the small perturbations, they are symmetric about the central strip of the CPW, thus the slotline (or even) mode of the CPW can hardly be excited, and the use of a bonding wire (or bridge) at the CPW-to-slotlines transition is not necessary in the above designs. Also, the transmission measurements have shown that the radiation losses introduced by the slotline sections are small and the radiations for the above small arrays are mainly from the microstrip patches. 


\section{EXPERIMENTAL RESULTS AND DISCUSSION}

Using design parameters obtained in the previous section, we fabricated on the FR4 substrate an $1 \times 2$ and an $1 \times 4$ arrays. The measured and simulated axial ratios and return losses of the $1 \times 2$ array are shown in the solid and dashed lines of Figs. 3 and 4, respectively. The $3 \mathrm{~dB}$ bandwidth of the axial ratio is about $0.9 \%$. A minimum axial ratio level of $0.73 \mathrm{~dB}$ is obtained at $4.02 \mathrm{GHz}$, while the minimum of return loss occurs at $4.03 \mathrm{GHz}$, which is close to the design frequency of 4.02 GHz. The discrepancy may be due to the mechanical tolerance exists in the test piece. The return loss remains below $-15 \mathrm{~dB}$ in the axial ratio bandwidth. The measured and simulated axial ratios and return losses of the $1 \times 4$ array are shown in the dotted and dash-dotted lines of Figs. 3 and 4, respectively. The $3 \mathrm{~dB}$ bandwidth of the axial ratio is about $0.9 \%$, as in the case of the $1 \times 2$ array. A minimum axial ratio level of $0.77 \mathrm{~dB}$ is obtained at $4.01 \mathrm{GHz}$, while the minimum of the return loss occurs at $4.02 \mathrm{GHz}$. The return loss remains below $-15 \mathrm{~dB}$ in the axial ratio bandwidth

The RHCP radiation patterns for the $1 \times 2$ and $1 \times 4$ arrays are measured and compared to the theoretical results in Fig. 5(a) and (b), respectively, at their respective minimum axial ratio frequencies of 4.02 and 4.01 GHz. The measured front-to-back ratios are $17.0 \mathrm{~dB}$ for the 1 $\times 2$ array and $17.3 \mathrm{~dB}$ for the $1 \times 4$ array. The theoretical directivities are 9.11 and $12.36 \mathrm{~dB}$ for the $1 \times 2$ and $1 \times 4$ arrays, respectively. They are calculated by using the approximate formula presented in [7]. The measured gains are 6.93 and $9.57 \mathrm{~dB}$ for the $1 \times 2$ and $1 \times 4$ arrays, respectively. From the theoretical directivities and the measured gains, the array efficiencies are calculated as $60.5 \%$ and $52.6 \%$ for the $1 \times 2$ and $1 \times 4$ arrays, respectively.

\section{CONCLUSION}

A simple T-shaped CPW-to-slotlines feeding structure has been used to design a circularly polarized linear patch antenna array. The validity of this design has been established by comparing with the experimental results on axial ratios, return losses, and radiation patterns of an $1 \times 2$ and an $1 \times 4$ arrays. The present design can be extended to form a 2-D array with good front-to-back ratio on a single substrate with only one straight CPW line.

\section{REFERENCES}

[1] X. H. Yang and W. X. Zhang, "Coplanar waveguide antenna arrays for MIC/MMIC at millimeter wave frequencies," Electron. Lett., vol. 26, no. 18, pp. 1464-1465, 1990.

[2] H. Kobayashi and Y. Yasuoka, "Slot-array antennas fed by coplanar waveguide for millimeter-wave radiation," IEEE Trans. Microwave Theory Tech., vol. 46, pp. 800-805, June 1998.

[3] T. F. Huang, S. W. Lu, and P. Hsu, "Analysis and design of coplanar waveguide-fed slot antenna array," IEEE Trans. Antennas Propagat., vol. 47, pp. 1560-1565, Oct. 1999.

[4] E. A. Soliman, S. Brebels, G. A. E. Vandenbosch, and E. Beyne, "Antenna arrays in MCM-D technology fed by coplanar CPW networks," IEEE Trans. Microwave Theory Tech., vol. 48, pp. 1065-1068, June 2000.

[5] J.-M. Laheurte, "Uniplanar monopulse antenna based on odd/even mode excitation of coplanar line," Electron. Lett., vol. 37, no. 6, pp. 338-340, 2001.

[6] S. Matsuzawa and K. Ito, "FDTD analysis of circularly polarized slot array antenna fed by coplanar waveguide," in IEEE AP-S Dig., 1997 pp. $1264-1267$

[7] E. A. Soliman, S. Brebels, E. Beyne, and G. A. E. Vandenbosch, " $2 \times$ 2 and $4 \times 4$ arrays of annular slot antennas in MCM-D technology fed by coplanar CPW networks," Proc. Inst. Elect. Eng.-Microw. Antennas Propag., vol. 146, no. 5, pp. 335-338, Oct. 1999.
[8] R. N. Simons, G. E. Ponchak, R. Q. Lee, and N. S. Fernandez, "Coplanar waveguide fed phased array antenna," in IEEE AP-S Dig., 1990, pp. $1778-1781$.

[9] E. T. Rahardjo, S. Kitao, and M. Haneishi, "Circularly polarized planar antenna excited by cross-slot coupled coplanar waveguide feedline," in IEEE AP-S Dig., 1994, pp. 2220-2223.

[10] H. Iwasaki, "A back-to-back rectangular-patch antenna fed by a CPW," IEEE Trans. Antennas Propagat., vol. 46, pp. 1527-1530, Oct. 1998.

[11] T. Ihara, T. Manabe, M. Fujita, and Y. Sugimoto, "Research activities on millimeter-wave indoor wireless communication systems at CRL," in Proc. ICUPC95, Tokyo, Japan, 1995, pp. 197-200.

[12] J. R. James, P. S. Hall, and C. Wood, Microstrip Antenna-Theory and Design. New York: Peregrinus, 1981, ch. 7.

\section{Singularities of Floquet Scattering Solution for Large Cell Spacings}

Hao Liu and Robert Paknys

\begin{abstract}
The Floquet moment method (FMM) is useful for computing the scattering by periodic structures composed of complex shaped cells. However, when the period $s$ is larger than $0.5 \lambda$, the computed field is singular at the grating lobe angles. In this paper, we demonstrate that these singularities have nothing to do with grating lobes, which are a physical phenomenon. Rather, they are a mathematical artifice of the FMM. This is demonstrated by modeling a flat ground plane with the FMM. We use a strip grating for the transverse magnetic case. The physical structure under investigation does not have any grating lobes, however the singular behavior can be made to appear and disappear by adjusting the cell spacing of the periodic structure.
\end{abstract}

Index Terms-Electromagnetic scattering, periodic structures.

\section{INTRODUCTION}

The Floquet solution is well-known to be useful in solving periodic structures, like phased array antennas [1] and frequency selective surface design [2]. In most of the applications, the spacing between cells is usually small compared with the wavelength in free space, where the Floquet solution gives good results. Recently [3], an attempt was made to apply the Floquet solution to periodic arrays with large cell spacings, and suspicious numerical results were found under some circumstances.

The simplest problem which has a periodic solution is a plane wave incident on a ground plane. In this paper, the field solution of a transverse magnetic (TM) plane wave incident on a conducting ground plane is investigated. The ground plane is divided into a periodic array of grating strips, and solved using Floquet modes. The Floquet solution for this periodic array of grating strips with various lengths is compared with the exact solution.

\section{Plane WaVe Incident on a Ground Plane}

Consider a $z$-polarized plane wave incident upon a conducting ground plane. The incident plane wave of unit amplitude is

$$
E_{z}^{i}(x, y)=e^{j k(x \cos \phi+y \sin \phi)}
$$

Manuscript received July 9, 2002; revised August 8, 2003.

The authors are with the Department of Electrical \& Computer Engineering, Concordia University, Montreal, QC H4B-1R6, Canada (e-mail: paknys@ece.concordia.ca).

Digital Object Identifier 10.1109/TAP.2004.829415 